International Mathematical Forum, 2, 2007, no. 62, 3089 - 3094

\title{
A Partial Multivariate Polynomial Interpolation Problem
}

\author{
E. Ballico ${ }^{1}$ \\ Dept. of Mathematics \\ University of Trento \\ 38050 Povo (TN), Italy \\ ballico@science.unitn.it
}

\begin{abstract}
Here we propose a partial multivariate polynomial interpolation problem in which we only prescribe some of the first order partial derivates along the coordinate axes.
\end{abstract}

Mathematics Subject Classification: 14N05; 15A72; 65D05

Keywords: polynomial interpolation; zero-dimensional scheme

\section{Introduction}

Fix an infinite field $K$, an integer $n>0$, and a system of coordinates $x_{1}, \ldots, x_{n}$ over the affine space $K^{n}$. Here we study the following polynomial interpolation problems in which we specify a genenal finite set $S \subset K^{n}$ and for each $P \in S$ we sprescribe the eavaluation at $P$ of a prescribed number of the $n$ partial derivatives $\partial / \partial_{x_{1}}, \ldots, \partial / \partial_{x_{n}}$. To state a polynomial interpolation problem we first need to fix a finite-dimensional $K$-vector space of the polynomial ring $K\left[x_{1}, \ldots, x_{n}\right]$. We fix an integer $d \geq 0$ and consider the $K$-vector space $H^{0}\left(\mathbf{P}^{n}, \mathcal{O}_{\mathbf{P}^{n}}(d)\right)$ of all $f \in K\left[x_{1}, \ldots, x_{n}\right]$ with total degree at most $d$. This vector space has dimension $\left(\begin{array}{c}n+d \\ n\end{array}\right)$. Now we describe our interpolation data. Fix $P \in K^{n}$, say $P=\left(b_{1}, \ldots, b_{n}\right)$ and an integer $c$ such that $0 \leq c \leq n$. If $c=0$, then set $Z(P, 0):=Z(P, 0 ; \emptyset)=\{P\}$. The scheme $Z(P, 0)$ correspond to the Lagrange interpolation problem with $P$ as its support: evaluate each $f \in K\left[x_{1}, \ldots, x_{n}\right]$ at $P$. Now assume $0<c \leq n$ and fix integers $1 \leq i_{1}<\cdots<$ $i_{c} \leq n$. Let $Z\left(P, c ; i_{1}, \ldots, i_{c}\right)$ denote the closed subscheme of $K^{n}$ defined by the $n$ equations: $x_{i}-b_{i}=0$, if $i \in\{1, \ldots, n\} \backslash\left\{i_{1}, \ldots, i_{c}\right\},\left(x_{i}-b_{i}\right)^{2}=0$ if $i \in$ $\left\{i_{1}, \ldots, i_{c}\right\}$. Hence $Z\left(P, c ; i_{1}, \ldots, i_{c}\right)$ is zero-dimensional, $Z\left(P, c ; i_{1}, \ldots, i_{c}\right)_{\text {red }}=$ $\{P\}$ and length $\left(Z\left(P, c ; i_{1}, \ldots, i_{c}\right)\right)=1+c . Z\left(P, c ; i_{1}, \ldots, i_{c}\right)$ define the following partial Hermite interpolation problem: given $f \in K\left[x_{1}, \ldots, x_{n}\right]$ evaluate at

\footnotetext{
${ }^{1}$ The author was partially supported by MIUR and GNSAGA of INdAM (Italy).
} 
$P$ the $c+1$ polynomials $f, \partial / \partial_{x_{i_{1}}}(f), \ldots, \partial / \partial_{x_{i_{c}}}(f)$. Abusing notations, we will write $Z(P, c)$ for any $Z\left(P, c ; j_{1}, \ldots, j_{c}\right) . Z(P, n)$ is the first infinitesimal neighborhood of $P$ in $K^{n}$. In characteristic zero the schemes $Z(P, n)$ are related to the dimensions of the Veroeose embeddings of $\mathbf{P}^{n}$ ([2], [6], [4]). We describe the difference between our interpolation problem and the one considered in [5]. The only difference is when $0<c<n$. M. C. Brambilla and G. Ottaviani took in [5] an arbitrary (and then a sufficiently general one) $c$-dimensional linear subspace of the $n$-dimensional vector space $T_{P} K^{n}$. Here we fix the coordinate system and for all $P$ we only allow linear subspaces spanned by $c$ of the axis.

Theorem 1. Assume char $(K) \neq 2$. Fix integers $n, d, x, c_{j}, 1 \leq j \leq x$ such that $n \geq 2, d \geq 3$, and $0 \leq c_{j} \leq n$ for all $j$. Fix a general $S \subset K^{n}$ such that $\sharp(S)=x$, say $S=\left\{P_{j}\right\}_{1 \leq j \leq x}$. Exclude the following cases:

(a) $n=2, d=4, x=5, c_{j}=2$ for all $j$;

(b) $n=3, d=4, x=9, c_{j}=3$ for $8 j$ 's and $c_{j}=2$ for one $j$;

(c) $n=3, d=4, x=9$ and $c_{j}=3$ for all $j$;

(d) $n=4, d=3, x=7$ and $c_{j}=4$ for all $j$;

(e) $n=4, d=4, x=14, c_{j}=4$ for all $j$.

For each $j$ such that $0<c_{j}<n$ there are $1 \leq i_{j, 1}<\cdots<i_{j, c_{j}} \leq n$ such that, setting $Z\left(P_{j}, c_{j}\right):=Z\left(P_{j}, c_{j} ; i_{j, 1}, \ldots, i_{j, c_{j}}\right)$ and $Z:=\cup_{j=1}^{x} Z\left(P_{j}, c_{j}\right)$, either $h^{0}\left(\mathbf{P}^{n}, \mathcal{I}_{Z}(d)\right)=0$ (case $\sum_{j=1}^{x)}\left(c_{j}+1\right) \geq\left(\begin{array}{c}n+d \\ n\end{array}\right)$ ) or $h^{1}\left(\mathbf{P}^{n}, \mathcal{I}_{Z}(d)\right)=0$ (case $\left.\sum_{j=1}^{x}\left(c_{j}+1\right) \leq\left(\begin{array}{c}n+d \\ n\end{array}\right)\right)$.

Notice that cases (a), ..., (e) in Theorem 1 are the exceptional cases of [4], Th. 1.1, i.e. making a finer polynomial interpolation problem we do not get more exceptional cases. Now we will see one case in which the corresponding statement is true for all choices of the integers $1 \leq i_{j, 1}<\cdots<i_{j, c_{j}} \leq n$. We prove the following result.

Theorem 2. Fix integers $x, d, c_{j}$ such that $x>0$ and $0 \leq c_{j} \leq 2$. Set $\alpha:=$ $\sharp\left(\left\{\left(c_{j}, i_{j}\right)=(1,1)\right\}\right.$ and $\beta:=\sharp\left(\left\{\left(c_{j}, i_{j}\right)=(1,2)\right\}\right.$. If char $(K) \neq 2$ assume $\alpha+\beta \geq d$. If $\operatorname{char}(K)=2$ assume $\min \{\alpha, \beta\} \geq\lceil(d+2) / 2\rceil$. For each integer $j$ such that $c_{j}=1$ fix any $i_{j} \in\{1,2\}$. Fix a general $S \subset K^{2}$ such that $\sharp(S)=x$ and order its points, say $S=\left\{P_{1}, \ldots, P_{x}\right\}$. Set $Z:=\cup_{j=1}^{x} Z\left(P_{j}, c_{j} ; i_{j}\right)$. Then either $h^{0}\left(\mathbf{P}^{2}, \mathcal{I}_{Z}(d)\right)=0$ or $h^{1}\left(\mathbf{P}^{2}, \mathcal{I}_{Z}(d)\right)=0$.

Inspired by [5], Th. 1.2, in the case $d=2$ we may prove the following result.

Theorem 3. Fix integers $n, x, c_{j}, 1 \leq j \leq x$ such that $n \geq 2,0 \leq c_{j} \leq n$ for all $j, c_{i} \geq c_{j}$ for all $i \leq j$ and $c_{j} \geq n+1-j$ for all $1 \leq j \leq \min \{x, n+1\}$. Set $c_{j}^{\prime}:=n+1-j$ for $1 \leq j \leq \min \{n+1, x\}$. If $x \geq n+2$ and $n+2 \leq j \leq x$, then take any $c_{j}^{\prime}$ with $0 \leq j \leq c_{j}^{\prime} \leq n$. Fix a general $S \subset K^{n}$ such that $\sharp(S)=x$, say $S=\left\{P_{j}\right\}_{1 \leq j \leq x}$. For each $j$ such that $0<c_{j}^{\prime}<n$ there are $1 \leq i_{j, 1}<$ $\cdots<i_{j, c_{j}^{\prime}} \leq n$ such that, setting $Z\left(P_{j}, c_{j}^{\prime}\right):=Z\left(P_{j}, c_{j}^{\prime} ; i_{j, 1}, \ldots, i_{j, c_{j}^{\prime}}\right)$ and $Z:=$ $\cup_{j=1}^{x} Z\left(P_{j}, c_{j}^{\prime}\right)$, either $h^{0}\left(\mathbf{P}^{n}, \mathcal{I}_{Z}(d)\right)=0($ case $x \geq n+1)$ or $h^{1}\left(\mathbf{P}^{n}, \mathcal{I}_{Z}(d)\right)=0$ 
(case $x \leq n+1)$. For all disjoint unions $W$ of $x$ schemes of type $Z\left(Q_{j}, c_{j}\right)$ we have $h^{0}\left(\mathbf{P}^{n}, \mathcal{I}_{W}(d)\right) \geq h^{0}\left(\mathbf{P}^{n}, \mathcal{I}_{Z}(d)\right)$ and $h^{1}\left(\mathbf{P}^{n}, \mathcal{I}_{W}(d)\right) \geq h^{1}\left(\mathbf{P}^{n}, \mathcal{I}_{Z}(d)\right)+$ $\sum_{j=1}^{y}\left(c_{j}-c_{j}^{\prime}\right)+\sum_{j=n+2}^{x}\left(1+c_{j}\right)$, where $y:=\min \{x, n+1\}$ and we take 0 instead of the last sum if $x \leq n+1$.

Question 1. What happens for higher order partial polynomial interpolation problems?

Concerning them, we only now how to handle (under very restrictive assumption) the case $n=2$ (as in the proof of Theorem 2.).

If $n \geq 2$, then $\mathbf{P}^{n}$ is not the only natural compactification of $K^{n}$. Assume $n \geq 2$ and fix integers $2 \leq s \leq n, m_{i}>0,1 \leq i \leq s, d_{i} \geq 0$, $1 \leq i \leq a$, such that $m_{1}+\cdots+m_{s}=n$. We take $\mathbf{P}^{m_{1}} \times \cdots \times \mathbf{P}^{m_{s}}$ as compactification of $K^{n}$. Instead of the $K$-vector space $H^{0}\left(\mathbf{P}^{n}, \mathcal{O}_{\mathbf{P}^{n}}(d)\right)$ we consider the linear subspace $H^{0}\left(\mathbf{P}^{m_{1}} \times \cdots \times \mathbf{P}^{m_{s}}, \mathcal{O}_{\mathbf{P}^{m_{1}} \times \cdots \times \mathbf{P}^{m_{s}}}\left(d_{1}, \ldots, d_{s}\right)\right)$ of all $f \in K\left[x_{1}, \ldots, x_{n}\right.$ such that in each monomial of $f$ appearing with non-zero coefficients the sum of the power of the variables $x_{1}, \ldots, x_{m_{1}}$ is at most $d_{1}$, of the variables $x_{m_{1}+1}, \ldots, x_{m_{1}+m_{2}}$ at most $d_{2}$, and so on. This vector space has dimension $\prod_{i=1}^{s}\left(\begin{array}{c}m_{i}+d_{i} \\ m_{i}\end{array}\right)$. Just a glance to the huge number of papers on the Segre-Veronese embeddings of projective spaces shows that in general this by far an harder problem. We only propose it.

\section{THE PROOFS}

Remark 1. Let $\bar{K}$ denote the algebraic closure of $K$. Since $K$ is infinite, $K^{n}$ is Zariski dense in $\bar{K}^{n}$. Hence the semicontinuity theorem for cohomology groups implies that it is sufficient to prove Theorems 1, 2 and 3 when $K$ is algebraically closed. From now on we will assume that $K$ is algebraically closed.

For any closed subscheme $Z$ of any quasi-projective scheme $A$ and every effective Cartier divisor $D$ of $A$ let $\operatorname{Res}_{D}(Z)$ denote the residual scheme of of $Z$ with respect to $D$, i.e. the closed subscheme of $A$ with $\mathcal{I}_{Z, A}: \mathcal{I}_{D, A}$ as its ideal sheaf. If $Z$ is zero-dimensional, then $\operatorname{length}(Z \cap D)+\operatorname{length}\left(\operatorname{Res}_{D}(Z)\right)=$ length $(Z)$. Hence if $A=K^{n}, D=\left\{x_{i}=\alpha\right\}$ is an affine coordinate hyperplane and $P \in D$, then $\operatorname{Res}_{D}(Z(P, 0))=\emptyset, \operatorname{Res}_{D}\left(Z\left(P, c ; i_{1}, \ldots, i_{c}\right)\right)=\{P\}$ if $i \in$ $\left\{i_{1}, \ldots, i_{c}\right\}$ and $\operatorname{Res}_{D}\left(Z\left(P, c ; i_{1}, \ldots, i_{c}\right)\right)=\emptyset$ if $i \notin\left\{i_{1}, \ldots, i_{c}\right\}$.

We will often use the following elementary form of the so-called Horace Lemma.

Lemma 1. Let $H \subset \mathbf{P}^{n}$ be a hyperplane and $Z \subset \mathbf{P}^{n}$ a closed subscheme. Then:

(a) $h^{0}\left(\mathbf{P}^{n}, \mathcal{I}_{Z}(d)\right) \leq h^{0}\left(\mathbf{P}^{n}, \mathcal{I}_{\operatorname{Res}_{H}(Z)}(d-1)\right)+h^{0}\left(H, \mathcal{I}_{Z \cap H}(d)\right)$;

(b) $h^{1}\left(\mathbf{P}^{n}, \mathcal{I}_{Z}(d)\right) \leq h^{1}\left(\mathbf{P}^{n}, \mathcal{I}_{\operatorname{Res}_{H}(Z)}(d-1)\right)+h^{1}\left(H, \mathcal{I}_{Z \cap H}(d)\right)$. 
Proof. By the very definition of residual scheme with respect to $H$, there is the following exact sequence

$$
0 \rightarrow \mathcal{I}_{\operatorname{Res}_{H}(Z)}(d-1) \rightarrow \mathcal{I}_{Z}(d) \rightarrow \mathcal{I}_{Z \cap H}(d) \rightarrow 0
$$

whose long cohomology exact sequence proves the lemma.

The following result (called the differential Horace lemma) is a very particular case of [3], Lemma 2.3 (see in particular Fig. 1 at p. 308).

Lemma 2. Assume char $(\mathbb{K}) \neq 2$. Let $H \subset \mathbf{P}^{n}$ be hyperplane, $Z \subset \mathbf{P}^{n}$ a closed subscheme not containing $H$ and $s$ a positive integer. Let $U$ be the union of $Z$ and $s$ general double points of $\mathbf{P}^{n}$. Let $S$ be the union of $s$ general points of $H$. Let $E \subset H$ be the union of $s$ general double points of $H$ (not double points of $\mathbf{P}^{n}$, i.e. each of them has length $\left.n\right)$. To prove $h^{1}\left(\mathbf{P}^{n}, \mathcal{I}_{U}(d)\right)=$ 0 (resp. $\left.h^{0}\left(\mathbf{P}^{n}, \mathcal{I}_{U}(d)\right)=0\right)$ it is sufficient to prove $h^{1}\left(H, \mathcal{I}_{(Z \cap H) \cup S}(d)\right)=$ $h^{1}\left(\mathbf{P}^{n}, \mathcal{I}_{\operatorname{Res}_{H}(Z) \cup E}(d-1)\right)=0\left(\operatorname{resp} . h^{0}\left(H, \mathcal{I}_{(Z \cap H) \cup S}(d)\right)=h^{0}\left(\mathbf{P}^{n}, \mathcal{I}_{\operatorname{Res}_{H}(Z) \cup E}(d-\right.\right.$ 1) $)=0$ ).

Proof of Theorem 1. The case $c_{j}=n$ for all $n$ is a famous theorem of Alexander and Hirschowitz ([1], [2], [6], [4]). Hence in the analysis of a few low degree cases we will assume $c_{j}<n$ for at least one index $j$. If Theorem 1 for the numerical datum $\left(n, d, x, c_{j}, 1 \leq j \leq x\right)$, then it is true for any numerical datum $\left(n, d, c_{j}^{\prime}, 1 \leq j \leq y\right)$ with $y>x, c_{j}^{\prime}=c_{j}$ for all $j \leq x$ and $c_{j}^{\prime}=0$ for all $j>x$. Hence in the analysis of a few low degree cases we will assume $c_{j}>0$ for all $j$. Let $H \subset \mathbf{P}^{n}$ be the completion of the hyperplane $\left\{x_{n}=0\right\}$. We have $\operatorname{length}(Z)=\sum_{j=1}^{x}\left(c_{j}+1\right)$.

(a) Assume $n=2$. First assume that there are at least $\lceil(d+1) / 2\rceil$ integers $c_{j}=2$. We fix $P_{1}, \ldots, P_{\lceil(d+1) / 2\rceil} \in H$. Set $Z_{1}:=\cup_{j=1}^{\lfloor(d+1) / 2\rfloor} Z\left(P_{j}, 2\right)$. Notice that $\operatorname{Res}_{H}\left(Z_{1}\right)=S_{1}:=\left\{P_{j}\right\}_{1 \leq j \leq\lfloor(d+1) / 2\rfloor}$. First assume $d+1$ even. Then length $\left(Z_{1} \cap\right.$ $H)=d+1$. Lemma 1 gives $h^{i}\left(\mathbf{P}^{2}, \mathcal{I}_{Z_{1}}(d)\right)=h^{i}\left(\mathbf{P}^{2}, \mathcal{I}_{S_{1}}(d-1)\right)$ for $i=0,1$. Now assume $d+1$ odd. We take $S_{1}, Z_{1}$ as above, but apply Lemma 2 with respect to the double point $2 P_{(d+2) / 2}$. We get that to check that the union $W$ of $Z_{1}$ with a general double point of $\mathbf{P}^{2}$ satisfies $h^{i}\left(\mathbf{P}^{2}, \mathcal{I}_{W}(d)\right)=0, i \in\{0,1\}$, it is sufficient to prove $h^{1}\left(\mathbf{P}^{2}, \mathcal{I}_{S_{1} \cup W^{\prime}}(d-1)\right)$, where $W^{\prime}:=Z\left(P_{(d+2) / 2}, 2 ; 2\right)$. We have length $\left(\left(S_{1} \cup W\right) \cap H\right)=d / 2+2 \leq d$ we equality only if $d=4$. We continue in this way adding double points with support on $D$ untill we exast all the double points. This proof just gave why (a) is the only case in which after finishing all the double point we do not come in the winning position. Then we continue adding schemes $Z(Q, 1 ; 2)$, except that at each step with a critical value $d^{\prime}$ we want to have a scheme with length exactly $d^{\prime}+1$ contained in $D$. If this is not possible for parity reasons, then we add a scheme $Z(Q, 1 ; 1)$ which intersects $D$ in a length 1 scheme, instead of a length 2 scheme.

(b) Here we assume $n \geq 3$ and $d=3$. We just claim that we may follow step by step [5], $\S 4$ (Lemma 4.2, Proposition ?? and then the proof of Theorem 4.1, taking at each step a coordinate linear subspace. It seems that the method 
of [5], §4, is a variation of the "méthode d'Horace éclaté " introduced in [1], but the main point of [5], $\S 4$ and $\S 5$, is to use it efficiently to check the many cases with critical $x$.

(c) Here we assume $n \geq 3$ and $d \geq 4$. Again, we just claim that it is sufficient to follow [5], §5. At this step, however, it seems possible also to mimick [6].

Proof of Theorem 2. We may (and will) assume that $K$ is algebraically closed. Since $K^{2}$ is Zariski dense in $\mathbf{P}^{2}$ we may freely switch from the affine plane to the projective plane. We assume $\operatorname{length}(Z) \leq\left(\begin{array}{c}d+2 \\ 2\end{array}\right)$, the other case being similar.

(a) Here we assume $\operatorname{char}(K) \neq 2$. Let $D \subset \mathbf{P}^{2}$ be a smooth plane conic. Since $\operatorname{char}(K) \neq 2$, there exactly two $\alpha \in K$ (resp. $\beta \in K$ ) such that the completion of the line $x_{1}=\alpha$ (resp. $x_{2}=\beta$ ) is tangent to $D$. Call $A_{1}, A_{2}$ (resp. $\left.B_{1}, B_{2}\right)$ be the contact points of these two lines with $D$. Since $\operatorname{deg}(D)=2$ and $D \cong \mathbf{P}^{1}, h^{1}\left(D, \mathcal{I}_{W, D}(t)\right)=h^{0}\left(D, \mathcal{I}_{W, D}(t)\right)=0$ for all zero-dimensional schemes $W \subset D$ with length $t+1$. First assume $c_{j}=2$ for at least $d+1$ indices $j$. We first put on $D$ the support of $d$ double points and use the differential Horace Lemma, i.e. Lemma 2 with respect to another point of $D$ and reduce to a similar situation for the integer $d^{\prime}:=d-2$ in which we also have $d$ general point and a general connected subscheme of $D$. Then we continue and go to the integer $d-4$ and so on. When we exaust all double points we insert the points with $c_{j}=1$. However, if we insert one of them at a non-tangential point, both its intersection with $D$ and its residue has length 1. Since $h^{0}\left(D, \mathcal{O}_{D}(t)\right)=2+h^{0}\left(D, \mathcal{O}_{D}(t-2)\right)$ it is sufficient to add at each step one of the points $A_{1}, A_{2}$ (case $i_{j}=1$ ) or one of the point $B_{1}, B_{2}$ (case $\left.i_{j}=2\right)$. The construction stops at integer $z \geq 1, z \equiv d(\bmod 2)$ either because we addedd $x$ connected components or because $t=2$. First assume that at the stopping value $z$ we had added $x$ connected component and call $M \subset D$ the residual scheme. Set $m:=\operatorname{length}(M)$. Te construction just given shows that we always have $m \leq z+4$. Hence if $z \geq 2$, there is no problem. Assume $z=1$. Since $\alpha+\beta \geq d$, we are sure that at this point we have no double point. Now assume that we stopped, having inserting at most $x-1$ connected components, say $y$ connected components, but that the remaining $x-y$ connected components are not enough to get a length $2 z+1$ scheme on $D$. Since $\alpha+\beta \geq d$, if $z \geq 2$ we may insert even the double points on $D$ and still have an $h^{1}$-vanishing, while if $z=1$ none of the $x-y$ components are double points. The configuration obtained on $D$ has $h^{1}=0$, because if not a line would contain all of it and its length would be at least 3 .

(b) Here we assume $\operatorname{char}(K)=2$. Without losing generality we may assume $\alpha \leq \beta$. Let $D \subset \mathbf{P}^{2}$ be the completion of the affine curve $\left\{x_{1}^{2}+x_{2}=0\right\}$. Thus $D$ is a smooth conic and each line $x_{1}=c, c \in K$ is tangent to $D$. No line $x_{2}=c^{\prime}$ is tangent to $D$. Notice that $Z(P, 1 ; 2) \subset D$ for all $P \in D$, while length $(Z(P, 1 ; 1) \cap D)=1$ for all $P \in D$. We copy the proof of part (a) with 
the following modifications. At no step we try to apply the differential Horace lemma. Instead, we insert a zero-dimensional scheme $Z\left(P_{j}, 1 ; 2\right)$ with $P_{j}$ a general point of $D$. At each step (say from degree $t$ to degree $t-2$ ) in which we do not have any added double point we insert at least one zero-dimensional scheme $Z\left(P_{j}, 1 ; 1\right)$, to be sure that the for the next step on $D$ the residual scheme has length at most $t+1-2=t-1$.

Proof of Theorem 3. Notice that the singular locus of a quadric hypersurface is a linear space. Set $y:=\min \{x, n+1\}$. Take any scheme $Z\left(P_{1}, n\right)$. Then take any $Z^{\prime}:=Z\left(P_{2}, n\right)$ or any $Z^{\prime \prime}:=Z\left(P_{2}, n-1\right), P_{2} \neq P_{1}$. We assume that $P_{2} \notin\left\langle Z^{\prime}\right\rangle$. We first get $h^{0}\left(\mathbf{P}^{n}, \mathcal{I}_{Z\left(P_{1}, n\right) \cup Z^{\prime \prime}}(2)\right)=\left(\begin{array}{c}n+2 \\ n\end{array}\right)-n-$ $1-n$ and $h^{1}\left(\mathbf{P}^{n}, \mathcal{I}_{Z\left(P_{1}, n\right) \cup Z^{\prime \prime}}(2)\right)=1$, because every element of $\left|\mathcal{I}_{Z\left(P_{1}, n\right) \cup Z^{\prime \prime}}\right|$ is singular at $P_{1}$ and $P_{2}$. Then our assumption on the coordinates used to describe $Z^{\prime}$ gives $h^{0}\left(\mathbf{P}^{n}, \mathcal{I}_{Z\left(P_{1}, n\right) \cup Z^{\prime}}(2)\right)=h^{0}\left(\mathbf{P}^{n}, \mathcal{I}_{Z\left(P_{1}, n\right) \cup Z^{\prime \prime}}(2)\right)$ and that every element of $\left|\mathcal{I}_{Z\left(P_{1}, n\right) \cup Z^{\prime \prime}}\right|$ is singular along $\left\langle\left\{P_{1}, P_{2}\right\}\right\rangle$. By assumption $\left\langle\left\{P_{1}, P_{2}\right\}\right\rangle$ is a line and we assume that it is a coordinate axis. Fix an integer $z$ such that $2 \leq z \leq \min \{x-1, n\}$ and assume defined $E:=\cup_{i=1}^{z} Z\left(P_{i}, c_{j}^{\prime}\right)$ so that $\left\langle\left\{P_{1}, \ldots, P_{z}\right\}\right\rangle$ is a coordinate $(z-1)$-dimensional linear subspace, $h^{1}\left(\mathbf{P}^{n}, \mathcal{I}_{E}(2)\right)=0, h^{0}\left(\mathbf{P}^{n}, \mathcal{I}_{E}(2)\right)=\left(\begin{array}{c}n+2 \\ 2\end{array}\right)-\sum_{i=1}^{z}(n+2-i)$ and $\left|\mathcal{I}_{E}(2)\right|$ is formed by all quadric cones whose vertex contains $\left\langle\left\{P_{1}, \ldots, P_{z}\right\}\right\rangle$. Fix $P_{z+1}$ so that $\left\langle\left\{P_{1}, \ldots, P_{z+1}\right\}\right\rangle$ is a $z$-dimensional coordinate linear system. There is a unique choice of the integers $1 \leq i_{1}<\cdots<i_{c_{z+1}^{\prime}} \leq n$ so that the union of $\left\langle\left\{P_{1}, \ldots, P_{z+1}\right\}\right\rangle$ and these coordinate lines spans $\mathbf{P}^{n}$. Choose these indices to define $Z\left(P_{z+1}, c_{z+1}^{\prime}\right.$ and set $F:=E \cup Z\left(P_{z+1}, c_{z+1}^{\prime}\right)$. As in the first step we get $h^{1}\left(\mathbf{P}^{n}, \mathcal{I}_{F}(2)\right)=0, h^{0}\left(\mathbf{P}^{n}, \mathcal{I}_{F}(2)\right)=\left(\begin{array}{c}n+2 \\ 2\end{array}\right)-\sum_{i=1}^{z+1}(n+2-i)$ and $\left|\mathcal{I}_{E}(2)\right|$ is formed by all quadric cones whose vertex contains $\left\langle\left\{P_{1}, \ldots, P_{z+1}\right\}\right\rangle$. And so on, proving all the assertions concerning $Z$. The other assertions follows from semicontinuity and [5], Th. 1.2.

\section{REFERENCES}

[1] J. Alexander, A. Hirschowitz, La méthode d'Horace éclaté: application à l'interpolation en degré quatre, Invent. Math. 107 (1992), 585-602.

[2] J. Alexander, A. Hirschowitz, Polynomial interpolation in several variables, J. Algebraic Geom. 4 (1995), 201-222.

[3] J. Alexander, A. Hirschowitz, An asymptotic vanishing theorem for generic unions of multiple points, Invent. Math. 140 (2000), 303-325.

[4] M. C. Brambilla and G. Ottaviani, On the Alexander-Hirschowitz theorem, arXiv:math/0701409.

[5] M. C. Brambilla and G. Ottaviani, On partial polynomial interpolation, arXiv:math/0705.4448.

[6] K. Chandler, A brief proof of a maximal rank theorem for generic double points in projective space, Trans. Amer. Math. Soc. 353 (2000), no. 5, 1907-1920.

Received: June 28, 2007 